\title{
Evaluación de la trayectoria investigadora a través de la distribución de citas: una aplicación a los Nobel de Economía ${ }^{1}$
}

\author{
María Isabel DORTA-GONZÁLEZ \\ Universidad de La Laguna \\ isadorta@ull.es \\ Pablo DORTA-GONZÁLEZ \\ Universidad de Las Palmas de Gran Canaria \\ pdorta@dmc.ulpgc.es
}

Recibido: mayo 2011

Aceptado: septiembre 2011

\begin{abstract}
Resumen: La distribución de citas de un investigador refleja el impacto de su producción y determina el éxito de su carrera profesional. Sin embargo, resulta difícil su aplicación por el carácter bidimensional. En los últimos años, han aparecido algunos indicadores que tratan de sintetizar, en un valor numérico, las características principales de esta distribución. A diferencia de otros indicadores, el índice h elimina los sesgos provocados por las colas de la distribución. Sin embargo, este indicador presenta limitaciones a la hora de discriminar entre investigadores con diferentes hábitos de publicación, penalizando a aquellos más selectivos, que no destacan por el número de publicaciones pero sí por el alto número de citas recibidas, frente a los grandes productores. En este trabajo se aplican dos nuevos indicadores en la evaluación de los ganadores del Nobel de Economía.
\end{abstract}

Palabras clave: índice h; análisis de citas; indicadores bibliométricos; evaluación científica; política investigadora.

\section{Evaluation of the research career through the citation distribution: An application to the Nobel Prizes in Economics}

\begin{abstract}
The citation distribution of a researcher reflects the impact of his production and determines the success of his scientific career. However, it is difficult to apply due to the bidimensional character. Some bibliometric indexes that try to synthesize with a numerical value the principal characteristics of this citation distribution have been proposed recently. In contrast with other bibliometric measures, the biases, that the distribution tails provoke, are eliminated by the h-index. On the other hand, some limitations to discriminate between researchers with different publication habits are presented. This index penalizes to selective researchers, distinguished by the number of citations instead of the number of publications, as compared to great

\footnotetext{
${ }^{1}$ Este trabajo ha sido financiado por el Ministerio de Ciencia e Innovación a través del proyecto ECO2008-05589.
} 
producers. In this work, two original indexes are applied to the evaluation of Nobel Prize winners in Economics.

Keywords: h-index; citation analysis; bibliometric indicators; scientific evaluation; research policy.

\section{INTRODUCCIÓN}

Los artículos son el resultado más directo de la investigación y aunque el juicio de expertos ha demostrado ser el método más apropiado para valorar su aportación al área, este sistema presenta algunas limitaciones, como el carácter subjetivo y su elevado coste. En este contexto, los indicadores bibliométricos representan indicios objetivos de calidad, que pueden complementar al juicio de expertos.

Existe un rechazo generalizado a evaluar la calidad de un artículo por el factor de impacto de la revista de publicación. Esto es debido a que incluso las mejores revistas publican trabajos de escaso éxito, y que algunos trabajos de gran éxito se publican en revistas que no están en los primeros puestos de los listados según el factor de impacto.

Por esta razón, los indicadores bibliométricos más empleados en la evaluación científica de los investigadores se basan en el recuento de las publicaciones y las citas recibidas. El número de publicaciones es un indicador cuantitativo que no valora la contribución al avance científico. Como indicadores cualitativos, capaces de valorar el impacto, influencia o visibilidad de la investigación, se suelen emplear el número total de citas y el promedio de citas por publicación.

Aunque estos indicadores reflejen en muchos casos el éxito de una carrera científica, en ocasiones, algunos trabajos acumulan un alto porcentaje del total de citas de un investigador. Además, las grandes colaboraciones introducen sesgos muy importantes, al recoger muchas citas pero derivadas del trabajo de un gran número de investigadores.

El índice h (Hirsch, 2005) trata de corregir estas limitaciones. Un investigador tiene un índice $\mathrm{h}$ cuando $\mathrm{h}$ de sus publicaciones han recibido al menos $\mathrm{h}$ citas, y el resto tiene $\mathrm{h}$ o menos citas. Este indicador estima el número de trabajos importantes, incrementando la exigencia a la vez que aumenta su valor. El número total de citas es generalmente mucho mayor que $\mathrm{h}^{2}$ (entre 3 y 5 veces según ha estimado Hirsch), ya que esta cantidad subestima las citas de los h artículos más citados e ignora los artículos con menos de h citas. Existe correlación entre el índice h y el éxito de un investigador apreciado por sus pares (Hirsch, 2005) y tiene, además, valor predictivo del éxito futuro (Hirsch, 2007).

El índice h ha sido extensamente estudiado (ver revisión de Bornmann y Daniel, 2007) y tiene importantes propiedades matemáticas (Glänzel, 2006). Sin embargo, también presenta limitaciones, algunas de las cuales se indican a continuación.

Depende del área y no es adecuado, por tanto, para comparar investigadores de diferentes áreas científicas, lo que se explica por los distintos hábitos de publicación, citación y colaboración según el campo. Este problema puede corregirse con 
el índice b (Bornmann y otros, 2007) que indica el número de artículos en el 10\% de los más citados en su campo, determinados a partir de los percentiles ISI-ESI, por ejemplo.

Correlaciona con el número de publicaciones y tiende a favorecer, por tanto, a aquellos que cuentan con carreras científicas más dilatadas (Cronin y Meho, 2006; Saad, 2006; Van-Raan, 2006). Para diferenciar entre investigadores activos e inactivos y poder comparar científicos en distintas etapas de su carrera, se ha propuesto la tasa de crecimiento (Rousseau y Ye, 2008). Otra alternativa es calcular el índice $\mathrm{h}$ para un determinado período de tiempo, como se hace en la aplicación empírica de este artículo, en lugar de toda la vida profesional de un investigador.

No considera todas las citas de los artículos más citados. Estos trabajos contribuyen al índice h, pero el número de veces que son citados no influye en su valor. Esto es debido a que se desprecian las colas de la distribución de citas. Estas colas corresponden a aquellas publicaciones que se alejan del impacto promedio, ya sea porque han sido muy citadas (cola superior), o porque apenas lo han sido (cola inferior). El índice g (Egghe, 2006) considera todas las citas de los g artículos más citados, y viene a representar un promedio de citas entre estos g artículos. Ordenados los artículos de forma decreciente del número de citas que reciben, $g$ es el mayor valor de forma que los primeros g artículos tienen, en su conjunto, al menos $\mathrm{g}^{2}$ citas. Sin embargo, como se ha indicado previamente, una cola superior pesada puede corresponder al trabajo de muchos autores en grandes líneas de investigación que generan muchas citas.

Finalmente, perjudica a los investigadores selectivos, aquellos con producción moderada pero de gran impacto, frente a los grandes productores, aquellos con una gran producción pero de impacto moderado. Aunque ha demostrado su utilidad para detectar a los investigadores más destacados en un área, existen evidencias empíricas de que no discrimina entre los situados en niveles intermedios. Surgen dudas especialmente en aquellos casos con valores similares de $\mathrm{h}$ en los que las curvas de citas se cortan, debido a que algunos investigadores presentan más altos niveles de citas al comienzo de la curva y más bajos al final. Además, no es consistente (Waltman y Van-Eck, 2011), debido a que el efecto de incorporar un nuevo trabajo con un número dado de citas puede ser diferente entre investigadores, incrementando el valor de $\mathrm{h}$ en unos casos y dejándolo igual en otros.

En este trabajo, se proponen dos complementos al índice h que aumentan su consistencia y favorecen a los autores más selectivos. Se presenta, además, una aplicación empírica al conjunto de ganadores del Nobel de Economía.

\section{INDICADORES CENTRALES PARA LA DISTRIBUCIÓN DE CITAS}

Dadas las p publicaciones de un autor en orden decreciente del número de citas, sean $\mathrm{C}_{\mathrm{i}}$ el número de citas recibidas por la publicación $\mathrm{i}, \mathrm{N}_{\mathrm{c}}=\sum_{\mathrm{i}=1}^{\mathrm{p}} \mathrm{c}_{\mathrm{i}}$ el número total de citas. 
Denominaremos distribución de citas a la representación del número de citas frente a la posición en la lista, y curva de citas a la línea que une estos puntos. El índice $h$ es el mayor número entero que cumple $c_{h} \geq h$. Gráficamente, es la parte entera del punto de intersección de la curva de citas con la bisectriz del primer cuadrante. Esto significa que el autor tiene $\mathrm{h}$ trabajos con al menos $\mathrm{h}$ citas cada uno.

El índice h proporciona una cota inferior $\mathrm{H}=\mathrm{h}^{2}$ del número total de citas y no incluye el volumen de citas en las colas de la distribución: cola superior, U, de artículos más citados y cola inferior, $\mathrm{L}$, de artículos menos citados. Se cumple que $\mathrm{N}_{\mathrm{c}}=\mathrm{H}+\mathrm{U}+\mathrm{L}$.

El peso relativo de las colas de la distribución de citas viene dado por $\mathrm{N}_{c} / \mathrm{H}$. De acuerdo a las estimaciones de Hirsch, si $\mathrm{N}_{c} / \mathrm{H}<3$ la distribución tiene colas poco pesadas, mientras que si $\mathrm{N}_{c} / \mathrm{H}>5$ la distribución tiene colas muy pesadas.

El índice $\mathrm{h}$ perjudica a aquellos investigadores que presentan colas pesadas. De manera especial, a aquellos con una relación de colas $U / L$ elevada. Cuando $U / L>1$, el peso de la cola superior es mayor que el de la cola inferior, lo que indica que se trata de un investigador selectivo. Cuando sucede lo contrario, esto es $\mathrm{U} / \mathrm{L}<1$, nos encontramos ante un investigador gran productor.

La Figura 1 muestra un ejemplo con dos curvas de citas. El primer investigador presenta niveles más altos de citas al comienzo de la curva y más bajos al final. Se aprecian, por tanto, dos perfiles muy diferentes, uno selectivo frente a otro más masivo en la publicación. Sin embargo, ambos presentan idéntico índice h. El índice $\mathrm{h}$ de un investigador puede incluso ser menor al del otro, aunque eso no tiene porqué indicar necesariamente que el primero presente una carrera con menos éxito. Esta gráfica refleja el problema de discriminar entre dos distribuciones con similares índices $\mathrm{h}$ pero con diferencias importantes en la relación entre las colas de la distribución.

Parece razonable, en estos casos, medir de alguna manera parte de U y L para complementar el índice h con las zonas próximas al cuadrado $\mathrm{H}$. De esta forma, se incrementa la capacidad de discriminación. Esta idea da lugar a los indicadores que se describen a continuación.

\subsection{INDICADOR DE ENTORNO}

Sea $\mathrm{E}$ (F) la zona superior (inferior) contigua a $\mathrm{H}$, esto es, aquella parte de la cola superior U (inferior L) de la distribución de citas más próxima a $\mathrm{H}$. La zona inferior corresponde a aquellos artículos que con mayor probabilidad pueden contribuir a incrementar el valor de $\mathrm{h}$ en el futuro, dado que están más próximos al núcleo que define su valor. La zona superior incluye aquellas citas que formarán parte del cuadrado $\mathrm{H}$ en el momento en que el índice $\mathrm{h}$ aumente de valor. Por ello, parece razonable incluir también dichas zonas y aumentar, de esta forma, el poder de discriminación del indicador.

Los indicadores de entorno consideran estas zonas contiguas (E y F) en cierto entorno de $\mathrm{h}$ de radio $\mathrm{j}$ de la forma: 


$$
H_{j}=(h-j) \cdot c_{h-j}+\sum_{i=h-j+1}^{h+j} c_{i}, j=1, \ldots, h-1 .
$$

$\mathrm{H}_{\mathrm{h}-1}$ incluye toda la cola superior $\mathrm{U}$. Aunque podría definirse el indicador también para otros valores mayores, hay que tener en cuenta que, en tal caso, sólo estaríamos añadiendo parte de la cola inferior.

La Figura 2 muestra el indicador de entorno de radio $\mathrm{h} / 2$ (parte entera) para una distribución de citas. Como se ha comentado anteriormente, aquellos autores cuyas distribuciones de citas presentan colas pesadas son perjudicados por el índice h. Sin embargo, para estos autores el indicador de entorno crece más rápidamente que para los investigadores con colas menos pesadas, aumentando el poder de discriminación.

Los investigadores selectivos también son perjudicados por el índice h. Sin embargo, el indicador de entorno corrige este problema. A modo de ejemplo, supongamos un investigador con 10 publicaciones, la menos citada de las cuales tiene 20 citas. En este caso $\mathrm{h}=10$ y representa tan sólo a 100 de las citas. Sin embargo, $\mathrm{H}_{1} \geq 200$, por lo que se ha doblado el volumen de citas representadas.

\subsection{INDICADOR DE INTERVALO}

El indicador de intervalo de radio $\mathrm{j}$ es la suma de las citas de los artículos en el intervalo $[h-j, h+j]$, esto es: $l_{j}=\sum_{i=h-j}^{h+j} c_{i}, j=1, \ldots, h-1$.

La Figura 2 muestra el indicador de intervalo de radio $\mathrm{h} / 2$ (parte entera) para una distribución de citas. Como puede observarse, el indicador de intervalo coincide con el área $\mathrm{G}+\mathrm{F}$, siendo $\mathrm{G}$ la zona izquierda y $\mathrm{F}$ la zona derecha contiguas a la recta $\mathrm{x}=\mathrm{h}$.

Puede observarse que en la cola inferior ambos indicadores coinciden. Sin embargo, en la cola superior existen diferencias significativas entre ambos indicadores. Mientras los indicadores de intervalo añaden secuencialmente las citas de los artículos situados a la izquierda del $\mathrm{h}$, los indicadores de entorno agregan áreas de tamaño variable.

En la aplicación empírica del apartado siguiente se determinará los radios del entorno e intervalo óptimos, es decir, el valor de j que mejor describe la parte central de la distribución de citas.

\section{APLICACIÓN EMPÍRICA}

Se estudia el comportamiento de los indicadores de entorno e intervalo en los 53 investigadores que han obtenido el Nobel de Economía. Los datos se han obtenido de la base de datos ISI-Web of Science en abril de 2010. 
Para estimar la capacidad de predicción de los indicadores a cinco años, y comparar con el índice h, se consideran los artículos que han sido citados y el número de citas obtenidas en dos instantes diferentes, $\mathrm{N}$ y N-5, siendo $\mathrm{N}$ el año de la concesión de dicho galardón. Se ha obtenido los indicadores $H_{j}, I_{j}, j=1, \ldots, h-1$, para $\mathrm{N}-5$ y se ha estimado empíricamente el valor de $\mathrm{j}$ (radio óptimo) que correlaciona mejor con los $H_{k}, I_{k}, k=j, \ldots, h-1$, en el instante $N$, es decir, los indicadores futuros.

La Tabla I muestra, para cada autor, el rango de publicaciones, con el primer y último año de artículos en la base de datos, el total de artículos que han sido citados y las citas totales en $\mathrm{N}$ y N-5. Esta tabla muestra también la evolución tanto del índice $\mathrm{h}$ como del cuadrado de citas $\mathrm{H}=\mathrm{h}^{2}$, en los instantes considerados, así como sus promedios. Como se comprobará con posterioridad, el índice h promedio guarda una estrecha relación con el radio del estimador óptimo.

A modo de ejemplo, la Figura 3 recoge las curvas de citas de seis investigadores de la muestra. Por claridad, se ha tomado 100 como valor máximo en cada eje. Para cada autor se muestran dos curvas, siendo la más próxima al origen de coordenadas la correspondiente a N-5. Esta representación permite observar gráficamente la evolución del índice h, así como distinguir entre investigadores selectivos y grandes productores. Como puede observarse, Becker, Engle y Kahneman presentan un comportamiento más selectivo que Akerlof, Granger y Buchanan, respectivamente.

La Figura 4 presenta los diagramas de dispersión producción-impacto para los 53 autores de la muestra. Como puede observarse, existe correlación lineal entre el número de artículos y el número de citas. Aquellos autores por encima de la recta de regresión muestran un comportamiento más selectivo que los situados por debajo de esta recta. Así, el autor más selectivo de la muestra es Kahneman mientras que el menos selectivo es Buchanan.

La Tabla II muestra los indicadores de entorno e intervalo para los dos períodos considerados. Aunque en el caso de algunos investigadores es posible calcular estos indicadores para un radio superior a doce, a medida que aumenta dicho radio se reduce el número de datos en cada columna de la tabla. Se ha optado por mostrar hasta el radio doce para garantizar con posterioridad que el coeficiente de correlación se calcule con al menos la mitad de los datos de la muestra.

Algunos de los mayores incrementos en el índice $\mathrm{h}$ se producen entre aquellos autores con un mayor ritmo de crecimiento en los indicadores de entorno en N-5. Por ejemplo, Kahneman pasa de $\mathrm{H}_{1}=1659$ a $\mathrm{H}_{12}=3163$ en el instante N-5. Este importante incremento adelanta la evolución del índice h, que aumenta 9 unidades en tan sólo cinco años. Algo similar ocurre también con Ostrom. Aunque se ha tomado como referencia los indicadores de entorno, en el caso de los indicadores de intervalo ocurre algo similar. 
Veamos algunos ejemplos donde se pone de manifiesto la capacidad de discriminación de los indicadores centrales frente al índice h. Aunque Buchanan presenta un índice h superior Kahneman en el período N-5, los indicadores de entorno de este último aumentan tan rápidamente que llega a tener un $\mathrm{H}_{12}$ superior al primero. Esto explica el mayor incremento en el h futuro (9 frente a 4). Algo similar sucede también con otros autores, como veremos a continuación.

Akerlof aumenta h en 2 unidades frente a Becker que lo hace en 4 y adelanta al primero según este indicador. Este hecho puede explicarse porque todos los indicadores centrales son mayores para el segundo autor, que es más selectivo que el primero atendiendo tanto a los indicadores centrales como al número total de artículos citados y citas recibidas. Algo similar ocurre también entre Engle y Granger. El primero adelanta al segundo en h, lo cual se corresponde con el hecho que todos los indicadores centrales del primero sean mayores a los del segundo.

Dado que los indicadores centrales representan un agregado de citas, su representación en función del radio es una función creciente. Esto puede observarse en la Figura 5, donde se muestra el comportamiento de estos indicadores para Kahneman y Buchanan, que como se ha indicado son los autores más y menos selectivos de la muestra, respectivamente. En la primera representación puede observarse cómo las líneas que unen los indicadores de entorno se cortan para ambos períodos. Inicialmente son mayores para Buchanan debido a que su índice h es mayor. Sin embargo, acaba superado por Kahneman dado que es más selectivo y, como se ha indicado, el incremento en los indicadores de entorno es entonces mayor. En la segunda representación puede observarse como las diferencias entre los indicadores de intervalo son menos significativas que en el caso anterior.

La Tabla III muestra los coeficientes de correlación lineal entre los indicadores. Como puede observarse, los indicadores de entorno para N-5 correlacionan fuertemente con los de N, por lo que parecen buenos estimadores a 5 años. En la mayor parte de los casos son superiores a 0.9 (un número significativo de ellos incluso superiores a 0.98 ). Las mayores correlaciones se sitúan próximas a la diagonal principal. La correlación promedio aumenta entre $\mathrm{H}_{1}$ y $\mathrm{H}_{6}$, donde se alcanza un máximo local. Aunque existen correlaciones promedio superiores (a partir de $\mathrm{H}_{9}$ ), en esos casos se emplean menos datos. Por tanto, el mejor estimador a cinco años es $\mathrm{H}_{6}$, cuyo radio es algo inferior a la mitad del índice $\mathrm{h}$ promedio de la muestra $(14.6 / 2=7.3)$.

Con el fin de poder apreciar qué indicadores ofrecen las mejores correlaciones, en la Tabla III también se muestran las diferencias entre las correlaciones para los indicadores de entorno e intervalo. Como puede observarse, todos los elementos de esta matriz son positivos, lo que significa que las correlaciones para los indicadores de entorno son mayores a los indicadores de intervalo.

Finalmente, la Tabla IV muestra para el período N-5 la relación entre el indicador de entorno óptimo y el rectángulo de citas de Hirsch. Esta medida es sólo comparable entre autores con valores similares de h. Dados varios autores con 
valores similares de $\mathrm{h}$, es más selectivo aquel para el que esta relación es mayor. A modo de ejemplo, Becker es más selectivo que Akerlof, Engle más que Granger, y Kahneman lo es más que Buchanan.

\section{CONCLUSIONES}

El índice h es un indicador bibliométrico que trata de medir el éxito de la carrera profesional de un investigador con una parte del volumen total de publicaciones y citas. El hecho de no considerar toda la producción e impacto, corrige los sesgos derivados de las grandes colaboraciones y los éxitos puntuales, que pueden no ser significativos en la carrera profesional en su conjunto. Sin embargo, distribuciones de citas muy diferentes, como las de un investigador selectivo y un gran productor, pueden dar lugar a similares índices $\mathrm{h}$.

En este trabajo se han propuesto dos complementos al índice h, el indicador de entorno y el de intervalo, con el objetivo de aumentar la capacidad de discriminación entre investigadores con similares $\mathrm{h}$ y mejorar la capacidad de predicción del éxito futuro. Estos indicadores consideran zonas que son mayores para los autores selectivos que para los grandes productores. De esta forma, se corrige uno de los problemas descritos en la literatura que indica que el índice $h$ beneficia a los grandes productores frente a aquellos más selectivos.

En la aplicación empírica se ha puesto de manifiesto que ambos indicadores centrales son buenos estimadores y que las correlaciones son mayores para el indicador de entorno. Además, se ha estimado empíricamente el radio que mejor describe la forma de la distribución de citas. Este radio óptimo es aproximadamente la mitad del índice h promedio de los individuos en evaluación.

\section{REFERENCIAS BIBLIOGRÁFICAS}

BORNMANN, L.; DANIEL, H.D. "What do we know about the h index?", Journal of the American Society for Information Science and Technology, 2007, 58/9: 13811385.

BORNMANN, L.; MUTZ, R.; DANIEL, H.D. "The $b$ index as a measure of scientific excellence. A promising supplement to the $\mathrm{h}$ index?", Cybermetrics, 2007, 11/1: paper 6.

CRONIN, B.; MEHO, L.I. "Using the h-index to rank influential information scientists", Journal of the American Society for Information Science and Technology, 2006, 57/9: 1275-1278.

EGGHE, L. "Theory and practise of the g-index", Scientometrics, 2006, 69/1: $131-152$.

GLÄNZEL, W. "On the h-index. A mathematical approach to a new measure of publication activity and citation impact", Scientometrics, 2006, 67/2: 315-321.

HIRSCH, J.E. "An index to quantify an individual's scientific research output", Proceedings of the National Academy of Sciences, 2005, 102/46: 16569-16572. 
HIRSCH, J.E. "Does the $\mathrm{h}$ index have predictive power?", Proceedings of the National Academy of Sciences, 2007, 104/49: 19193-19198.

ROUSSEAU, R.; Ye, F.Y. "A proposal for a dynamic h-type index", Journal of the American Society for Information Science and Technology, 2008, 59/11: 1853-1855.

SAAD, G. "Exploring the h-index at the author and journal levels using bibliometric data of productive consumer scholars and business-related journals respectively", SCIENTOMETRICS, 2006, 69/1: 117-120.

VAN-Raan, A.F.J. "Comparisons of the Hirsch-index with standard bibliometric indicators and with peer judgment for 147 chemistry research groups", Scientometrics, 2006, 67/3: 491-502.

WALTMAN, L.; Van-Eck, N.J. "The inconsistency of the h-index", Journal of the American Society for Information Science and Technology, 2011, in press.

\section{FIGURAS}

Figura 1. Dos curvas de citas que se cortan, con idéntico índice h y número total de citas, pero diferente promedio de citas por artículo.

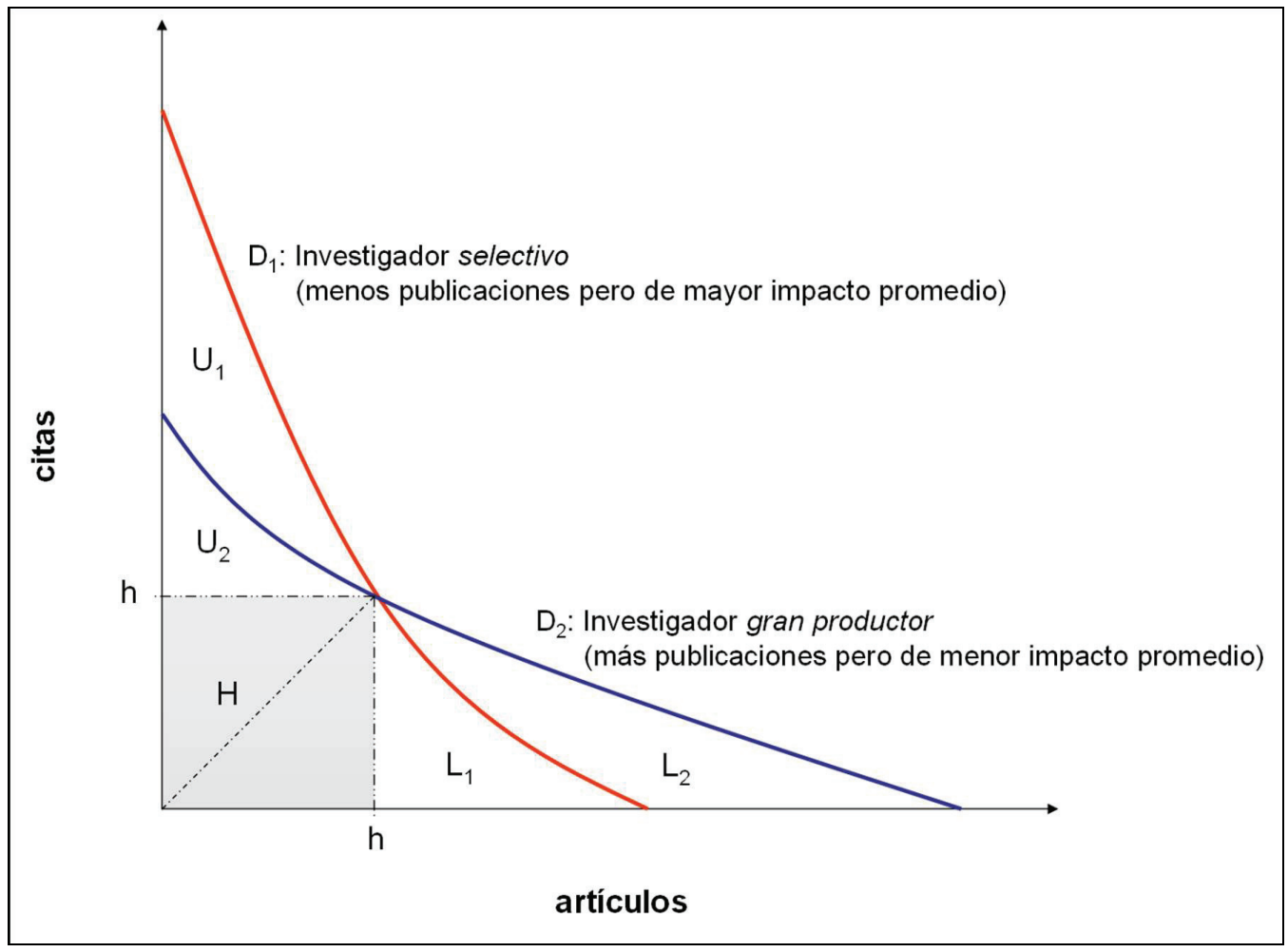


María Isabel Dorta-González, Pablo Dorta-González Evaluación de la trayectoria investigadora...

Figura 2. Indicador de entorno (a) e intervalo (b) de radio $\mathrm{j}=\mathrm{h} / 2$.

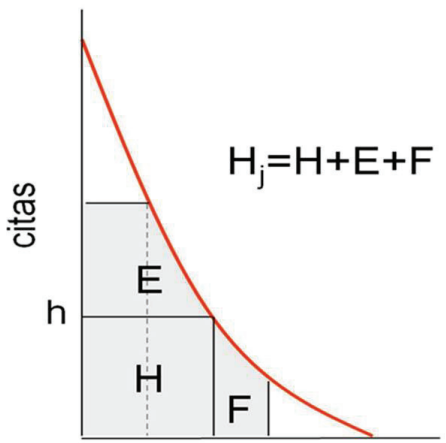

$\mathrm{h} / 2 \mathrm{~h} \quad 3 \mathrm{~h} / 2$

(a)

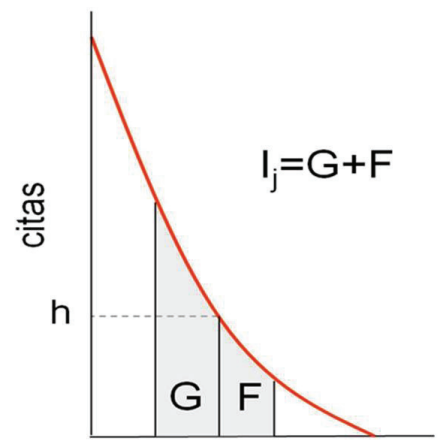

$\mathrm{h} / 2 \mathrm{~h} \quad 3 \mathrm{~h} / 2$

artículos

(b)

Figura 3. Curvas de citas para seis investigadores de la muestra en N-5 y N.
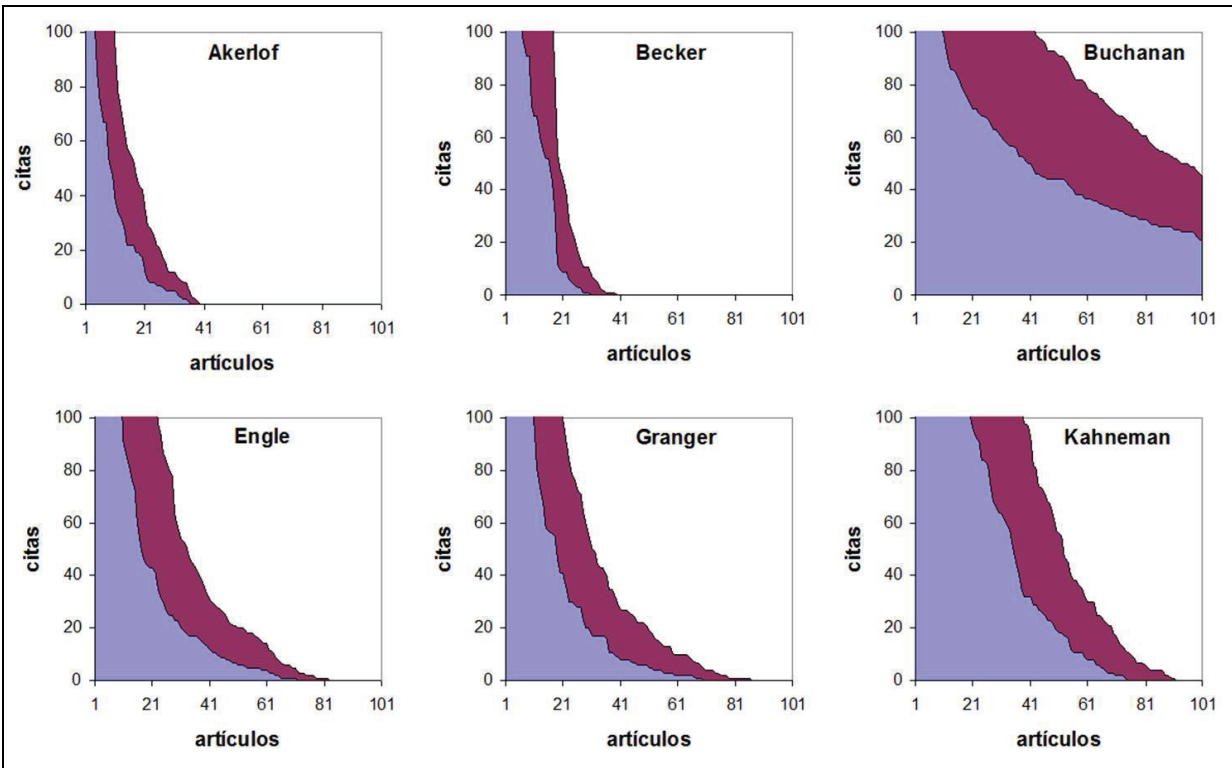
María Isabel Dorta-González, Pablo Dorta-González Evaluación de la trayectoria investigadora...

Figura 4. Diagramas de dispersión producción-impacto.

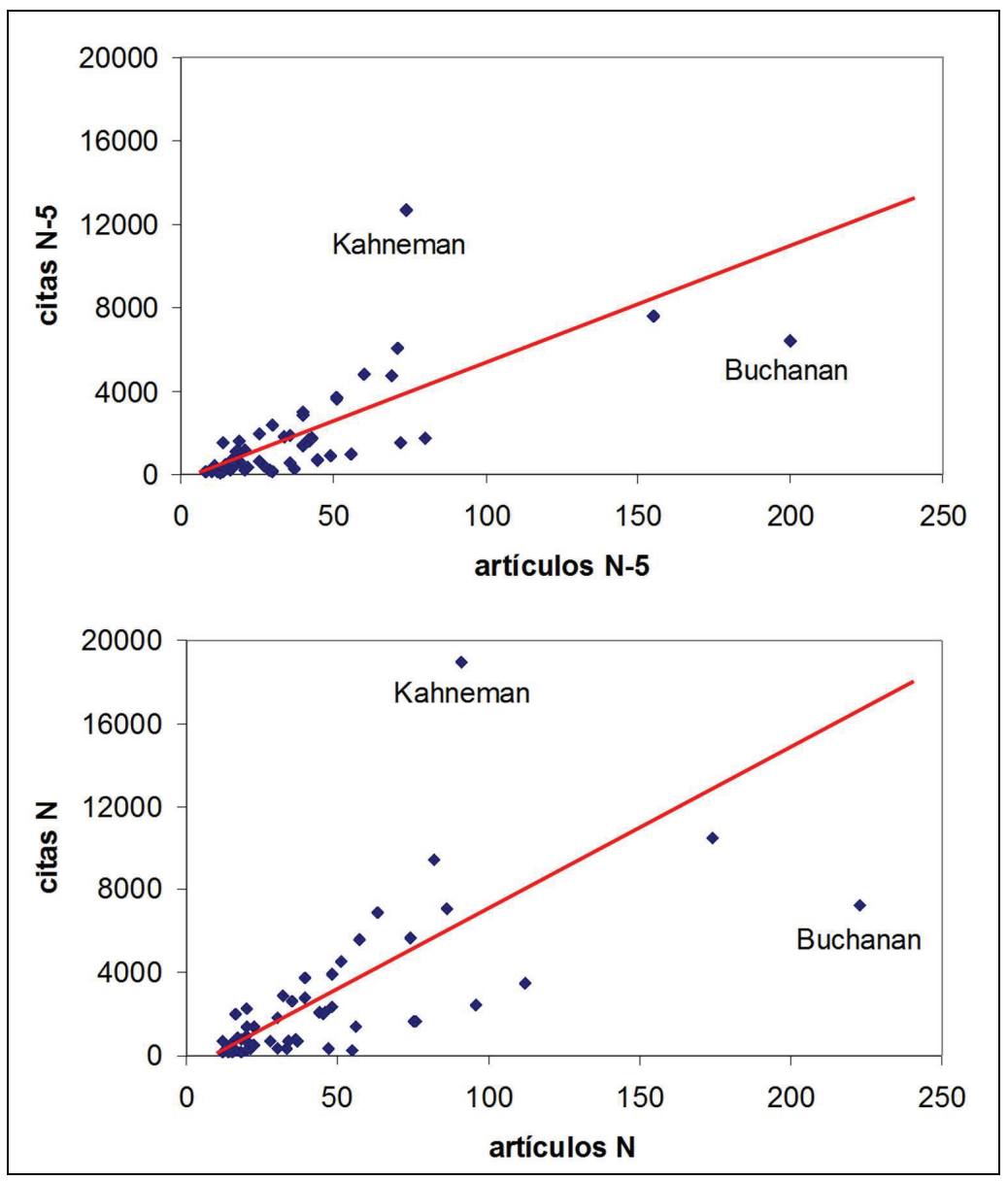


María Isabel Dorta-González, Pablo Dorta-González Evaluación de la trayectoria investigadora ...

Figura 5. Indicadores de entorno e intervalo de dos autores de la muestra.

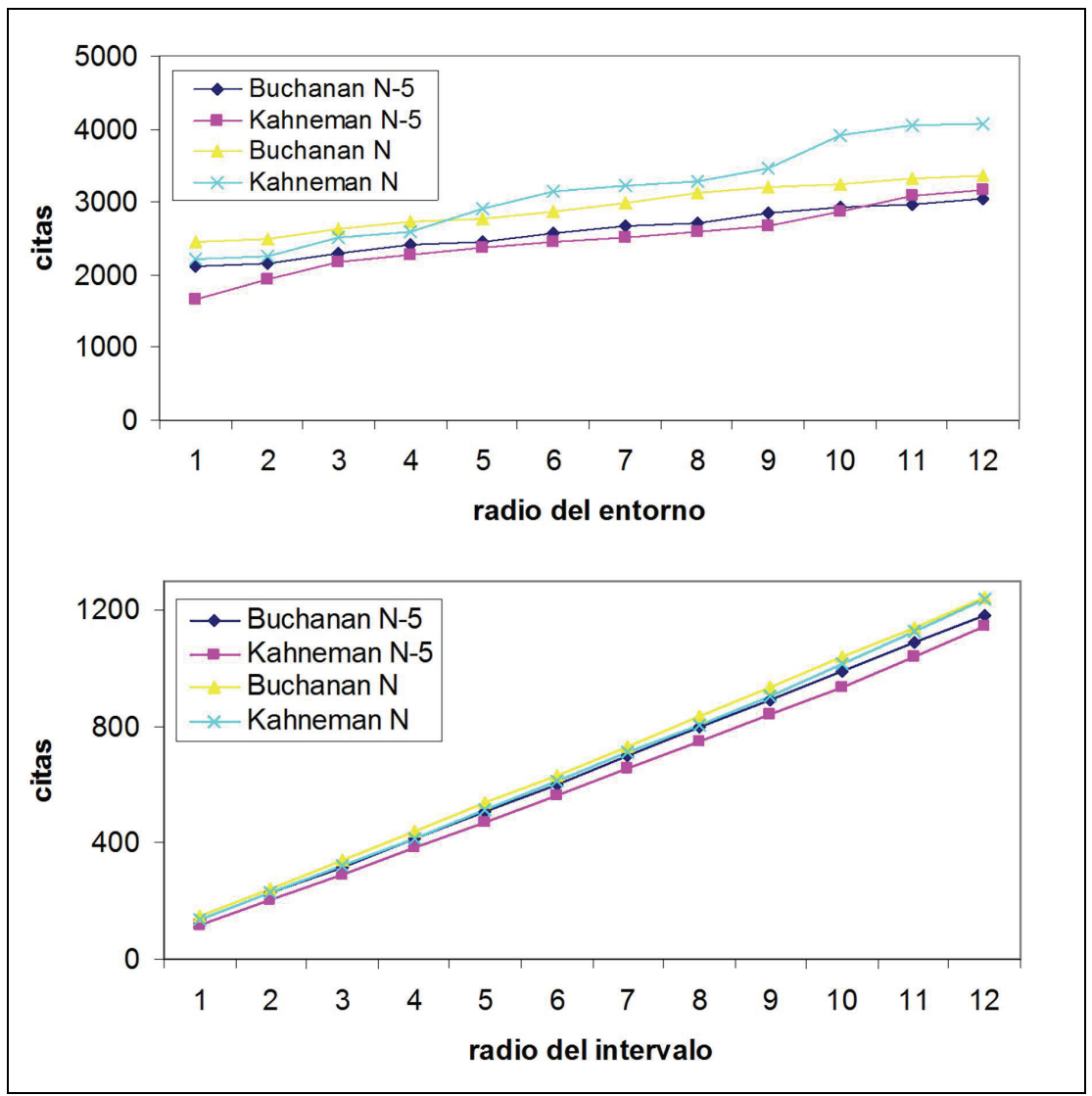




\section{TABLAS}

Tabla I. Producción, impacto e índices para los autores de la muestra.

\begin{tabular}{|c|c|c|c|c|c|c|c|c|c|c|c|c|}
\hline & \multirow[b]{2}{*}{ Autor } & \multirow{2}{*}{$\begin{array}{c}\text { Rango } \\
\text { artículos ISI } \\
\text { [primero, último] }\end{array}$} & \multirow{2}{*}{$\begin{array}{l}\text { Año } \\
\text { Nobel } \\
\text { N }\end{array}$} & \multicolumn{2}{|c|}{$\begin{array}{l}\text { Artículos } \\
\text { con citas }\end{array}$} & \multicolumn{2}{|c|}{ Citas } & \multicolumn{3}{|c|}{ Índice $\mathrm{h}$} & \multicolumn{2}{|c|}{$\mathrm{H}=\mathrm{h}^{2}$} \\
\hline & & & & N-5 & $\mathrm{N}$ & N-5 & $\mathrm{N}$ & $\mathrm{N}-5$ & $\mathrm{~N}$ & $\Delta \mathrm{h}$ & N-5 & $\mathrm{N}$ \\
\hline 1 & Akerlof, GA & {$[1969,2008]$} & 2001 & 36 & 39 & 1884 & 2805 & 19 & 21 & 2 & 361 & 441 \\
\hline 2 & Allais, $\mathrm{M}$ & [1949. 2000] & 1988 & 13 & 20 & 169 & 256 & 4 & 4 & 0 & 16 & 16 \\
\hline 3 & Arrow, KJ & {$[1951,2009]$} & 1972 & 22 & 34 & 327 & 727 & 10 & 14 & 4 & 100 & 196 \\
\hline 4 & Aumann, RJ & {$[1956,2010]$} & 2005 & 43 & 48 & 1769 & 2382 & 16 & 18 & 2 & 256 & 324 \\
\hline 5 & Becker, GS & {$[1957,2007]$} & 1992 & 30 & 39 & 2368 & 3743 & 18 & 22 & 4 & 324 & 484 \\
\hline 6 & Buchanan, JM & {$[1941,2008]$} & 1986 & 200 & 223 & 6413 & 7292 & 45 & 49 & 4 & 2025 & 2401 \\
\hline 7 & Coase, RH & {$[1959,2000]$} & 1991 & 17 & 20 & 656 & 977 & 6 & 8 & 2 & 36 & 64 \\
\hline 8 & Debreu, G & {$[1951,1999]$} & 1983 & 20 & 20 & 476 & 735 & 12 & 14 & 2 & 144 & 196 \\
\hline 9 & Engle, RF & {$[1972,2008]$} & 2003 & 71 & 82 & 6093 & 9469 & 26 & 33 & 7 & 676 & 1089 \\
\hline 0 & Fogel, RW & {$[1962,2005]$} & 1993 & 16 & 16 & 204 & 241 & 9 & 9 & 0 & 81 & 81 \\
\hline 11 & Friedman, M & {$[1941,2008]$} & 1976 & 14 & 21 & 118 & 362 & 4 & 8 & 4 & 16 & 64 \\
\hline 12 & Granger, CWJ & {$[1957,2009]$} & 2003 & 69 & 86 & 4734 & 7104 & 27 & 32 & 5 & 729 & 1024 \\
\hline 13 & Haavelmo, T & {$[1938,2007]$} & 1989 & 12 & 14 & 166 & 199 & 6 & 6 & 0 & 36 & 36 \\
\hline 14 & Harsanyi, JC & {$[1956,1998]$} & 1994 & 36 & 37 & 574 & 692 & 12 & 13 & 1 & 144 & 169 \\
\hline 15 & Heckman, JJ & {$[1974,2009]$} & 2000 & 51 & 74 & 3687 & 5699 & 23 & 30 & 7 & 529 & 900 \\
\hline 16 & Hurwicz, L & {$[1946,2003]$} & 2007 & 26 & 28 & 598 & 704 & 10 & 11 & 1 & 100 & 121 \\
\hline 17 & Kahneman, D & {$[1963,2008]$} & 2002 & 74 & 91 & 12682 & 18965 & 37 & 46 & 9 & 1369 & 2116 \\
\hline 8 & Klein, LR & {$[1943,2009]$} & 1980 & 37 & 47 & 260 & 378 & 9 & 10 & 1 & 81 & 100 \\
\hline 19 & Koopmans, TC & {$[1949,1982]$} & 1975 & 13 & 15 & 91 & 166 & 6 & 8 & 2 & 36 & 64 \\
\hline 0 & Krugman, PR & {$[1976,2008]$} & 2008 & 51 & 57 & 3615 & 5566 & 29 & 34 & 5 & 841 & 1156 \\
\hline 1 & Kydland, FE & {$[1976,2007]$} & 2004 & 14 & 16 & 1505 & 2043 & 11 & 12 & 1 & 121 & 144 \\
\hline 22 & Leontief, W & {$[1936,1998]$} & 1973 & 13 & 18 & 59 & 150 & 5 & 8 & 3 & 25 & 64 \\
\hline 3 & Lucas, RE & {$[1962,2009]$} & 1995 & 40 & 51 & 2986 & 4507 & 24 & 29 & 5 & 576 & 841 \\
\hline 24 & Maskin, ES & {$[1983,2008]$} & 2007 & 10 & 12 & 120 & 173 & 7 & 8 & 1 & 49 & 64 \\
\hline 25 & McFadden, DL & {$[1963,2007]$} & 2000 & 11 & 14 & 266 & 376 & 8 & 9 & 1 & 64 & 81 \\
\hline 6 & Merton, RC & {$[1969,2006]$} & 1997 & 26 & 32 & 1976 & 2874 & 18 & 19 & 1 & 324 & 361 \\
\hline 27 & Miller, MH & {$[1956,1999]$} & 1990 & 21 & 30 & 1177 & 1809 & 11 & 14 & 3 & 121 & 196 \\
\hline 28 & Mirrlees, JA & {$[1962,1999]$} & 1996 & 19 & 22 & 1139 & 1428 & 13 & 14 & 1 & 169 & 196 \\
\hline 9 & Modigliani, F & {$[1944,2004]$} & 1985 & 40 & 45 & 1362 & 2008 & 16 & 19 & 3 & 256 & 361 \\
\hline 30 & Mundell, RA & {$[1957,2009]$} & 1999 & 18 & 18 & 600 & 754 & 10 & 10 & 0 & 100 & 100 \\
\hline 31 & Myerson, RB & {$[1977,2009]$} & 2007 & 34 & 35 & 1805 & 2657 & 15 & 17 & 2 & 225 & 289 \\
\hline 2 & Nash, JF & {$[1950,2009]$} & 1994 & 49 & 75 & 901 & 1655 & 13 & 20 & 7 & 169 & 400 \\
\hline 33 & North, DC & {$[1956,2009]$} & 1993 & 21 & 30 & 222 & 350 & 10 & 12 & 2 & 100 & 144 \\
\hline 34 & Ostrom, E & {$[1965,2010]$} & 2009 & 72 & 112 & 1547 & 3537 & 22 & 31 & 9 & 484 & 961 \\
\hline 35 & Phelps, ES & {$[1961,2009]$} & 2006 & 42 & 44 & 1664 & 2083 & 17 & 19 & 2 & 289 & 361 \\
\hline
\end{tabular}


María Isabel Dorta-González, Pablo Dorta-González Evaluación de la trayectoria investigadora...

\begin{tabular}{|c|c|c|c|c|c|c|c|c|c|c|c|c|}
\hline & \multirow[b]{2}{*}{ Autor } & \multirow{2}{*}{$\begin{array}{c}\text { Rango } \\
\text { artículos ISI } \\
\text { [primero, último] }\end{array}$} & \multirow{2}{*}{$\begin{array}{c}\text { Año } \\
\text { Nobel } \\
\text { N }\end{array}$} & \multicolumn{2}{|c|}{$\begin{array}{l}\text { Artículos } \\
\text { con citas }\end{array}$} & \multicolumn{2}{|c|}{ Citas } & \multicolumn{3}{|c|}{ Índice $\mathrm{h}$} & \multicolumn{2}{|c|}{$\mathrm{H}=\mathrm{h}^{2}$} \\
\hline & & & & N-5 & $\mathrm{N}$ & N-5 & $\mathrm{N}$ & N-5 & $\mathrm{N}$ & $\Delta \mathrm{h}$ & N-5 & $\mathrm{N}$ \\
\hline \multirow[t]{2}{*}{36} & Prescott, EC & {$[1968,2009]$} & 2004 & 40 & 48 & 2833 & 3926 & 19 & 24 & 5 & 361 & 576 \\
\hline & Samuelson, PA & {$[1938,2010]$} & 1970 & 30 & 55 & 124 & 283 & 6 & 9 & 3 & 36 & 81 \\
\hline \multirow[t]{2}{*}{38} & Schelling, TC & {$[1956,2000]$} & 2005 & 11 & 12 & 413 & 680 & 9 & 9 & 0 & 81 & 81 \\
\hline & Scholes, MS & {$[1970,2000]$} & 1997 & 19 & 20 & 1604 & 2231 & 10 & 11 & 1 & 100 & 121 \\
\hline \multirow[t]{2}{*}{40} & Schultz, TW & {$[1932,1992]$} & 1979 & 29 & 33 & 230 & 342 & 7 & 9 & 2 & 49 & 81 \\
\hline & Selten, $\mathrm{R}$ & {$[1962,2009]$} & 1994 & 8 & 14 & 122 & 299 & 5 & 9 & 4 & 25 & 81 \\
\hline 42 & Sen, AK & {$[1957,1997]$} & 1998 & 15 & 15 & 488 & 575 & 8 & 8 & 0 & 64 & 64 \\
\hline 3 & Sharpe, WF & {$[1963,2008]$} & 1990 & 18 & 20 & 1103 & 1416 & 7 & 8 & 1 & 49 & 64 \\
\hline 44 & Simon, HA & {$[1945,2001]$} & 1978 & 56 & 76 & 982 & 1643 & 16 & 21 & 5 & 256 & 441 \\
\hline 5 & Smith, VL & {$[1957,2007]$} & 2002 & 80 & 96 & 1719 & 2419 & 22 & 26 & 4 & 484 & 676 \\
\hline 46 & Solow, RM & {$[1952,2005]$} & 1987 & 42 & 46 & 1636 & 2129 & 18 & 21 & 3 & 324 & 441 \\
\hline 7 & Spence, $\mathrm{M}$ & {$[1973,2001]$} & 2001 & 17 & 17 & 734 & 915 & 11 & 11 & 0 & 121 & 121 \\
\hline 48 & Stigler, GJ & {$[1937,1995]$} & 1982 & 45 & 56 & 695 & 1413 & 13 & 17 & 4 & 169 & 289 \\
\hline 9 & Stiglitz, JE & {$[1966,2009]$} & 2001 & 155 & 174 & 7618 & 10459 & 44 & 50 & 6 & 1936 & 2500 \\
\hline 50 & Stone, $\mathrm{R}$ & {$[1956,1991]$} & 1984 & 14 & 18 & 120 & 145 & 7 & 7 & 0 & 49 & 49 \\
\hline 1 & Tobin, J & {$[1950,2005]$} & 1981 & 27 & 36 & 428 & 812 & 10 & 14 & 4 & 100 & 196 \\
\hline 52 & Vickrey, W & {$[1941,1998]$} & 1996 & 17 & 22 & 329 & 481 & 6 & 7 & 1 & 36 & 49 \\
\hline \multirow[t]{2}{*}{3} & Williamson, OE & {$[1963,2008]$} & 2009 & 60 & 63 & 4793 & 6912 & 26 & 29 & 3 & 676 & 841 \\
\hline & Media & & & 37,1 & 45 & 1701,6 & 2489,5 & 14,6 & 17,4 & & 299,8 & 421,8 \\
\hline
\end{tabular}


Tabla II. Indicadores de entorno e intervalo para N-5 y N (hasta radio doce).

\begin{tabular}{|c|c|c|c|c|c|c|c|c|c|c|c|c|c|}
\hline \multirow[b]{2}{*}{ Autor } & \multicolumn{13}{|c|}{ N-5 } \\
\hline & $\mathrm{H}_{1}$ & $\mathrm{H}_{2}$ & $\mathrm{H}_{3}$ & $\mathrm{H}_{4}$ & $\mathrm{H}_{5}$ & $\mathrm{H}_{6}$ & $\mathrm{H}_{7}$ & $\mathrm{H}_{8}$ & $\mathrm{H}_{9}$ & $\mathrm{H}_{10}$ & $\mathrm{H}_{11}$ & $\mathrm{H}_{12}$ & $\mathrm{I}_{1}$ \\
\hline Akerlof, GA & 378 & 441 & 450 & 458 & 550 & 609 & 640 & 690 & 785 & 844 & 961 & 966 & 55 \\
\hline Allais, M & 97 & 120 & 162 & - & - & - & - & - & - & - & - & - & 41 \\
\hline Arrow, KJ & 127 & 159 & 178 & 213 & 222 & 253 & 271 & 297 & 322 & - & - & - & 31 \\
\hline Aumann, RJ & 379 & 446 & 470 & 493 & 624 & 654 & 763 & 809 & 857 & 1085 & 1460 & 1496 & 57 \\
\hline Becker, GS & 775 & 929 & 938 & 989 & 1047 & 1160 & 1164 & 1207 & 1381 & 1382 & 1432 & 1517 & 87 \\
\hline Buchanan, JM & 2114 & 2158 & 2286 & 2412 & 2456 & 2578 & 2660 & 2704 & 2854 & 2930 & 2970 & 3041 & 136 \\
\hline Coase, $\mathrm{RH}$ & 95 & 121 & 135 & 168 & 647 & - & - & - & - & - & - & - & 31 \\
\hline Debreu, G & 166 & 216 & 234 & 238 & 248 & 311 & 338 & 356 & 467 & 475 & 476 & - & 36 \\
\hline Engle, RF & 801 & 874 & 966 & 1121 & 1183 & 1202 & 1239 & 1292 & 1377 & 1554 & 1765 & 1822 & 81 \\
\hline Fogel, RW & 98 & 111 & 129 & 134 & 162 & 198 & 204 & 204 & - & - & - & - & 28 \\
\hline Friedman, M & 53 & 61 & 104 & - & - & - & - & - & - & - & - & - & 25 \\
\hline Granger, CWJ & 779 & 824 & 868 & 885 & 1012 & 1155 & 1172 & 1284 & 1462 & 1490 & 1517 & 1542 & 79 \\
\hline Haavelmo, T & 45 & 89 & 93 & 130 & 165 & - & - & - & - & - & - & - & 17 \\
\hline Harsanyi, JC & 201 & 222 & 250 & 308 & 352 & 372 & 390 & 412 & 442 & 534 & 547 & - & 41 \\
\hline Heckman, JJ & 690 & 733 & 772 & 961 & 1069 & 1084 & 1114 & 1142 & 1168 & 1439 & 1449 & 1514 & 81 \\
\hline Hurwicz, L & 190 & 198 & 241 & 247 & 253 & 478 & 519 & 563 & 582 & - & - & - & 38 \\
\hline Kahneman, D & 1659 & 1936 & 2172 & 2270 & 2363 & 2454 & 2511 & 2595 & 2676 & 2861 & 3092 & 3163 & 119 \\
\hline Klein, LR & 114 & 129 & 136 & 157 & 179 & 188 & 202 & 217 & - & - & - & - & 30 \\
\hline Koopmans, TC & 51 & 56 & 66 & 82 & 89 & - & - & - & - & - & - & - & 19 \\
\hline Krugman, PR & 979 & 1112 & 1134 & 1206 & 1274 & 1339 & 1358 & 1418 & 1476 & 1492 & 1633 & 1664 & 88 \\
\hline Kydland, FE & 163 & 184 & 257 & 285 & 303 & 328 & 348 & 474 & 1424 & 1505 & - & - & 37 \\
\hline Leontief, W & 33 & 38 & 49 & 55 & - & - & - & - & - & - & - & - & 15 \\
\hline Lucas, RE & 670 & 716 & 760 & 902 & 943 & 995 & 1024 & 1036 & 1090 & 1138 & 1208 & 1237 & 76 \\
\hline Maskin, ES & 59 & 72 & 102 & 114 & 114 & 120 & - & - & - & - & - & - & 19 \\
\hline McFadden, DL & 104 & 146 & 161 & 173 & 179 & 219 & 266 & - & - & - & - & - & 32 \\
\hline Merton, RC & 445 & 492 & 550 & 574 & 584 & 627 & 697 & 748 & 838 & 846 & 1112 & 1166 & 61 \\
\hline Miller, MH & 153 & 171 & 179 & 235 & 541 & 600 & 697 & 860 & 865 & 1177 & - & - & 36 \\
\hline Mirrlees, JA & 231 & 250 & 298 & 315 & 336 & 575 & 635 & 670 & 734 & 1088 & 1122 & 1139 & 44 \\
\hline Modigliani, F & 455 & 498 & 525 & 549 & 637 & 678 & 742 & 766 & 822 & 877 & 1052 & 1069 & 63 \\
\hline Mundell, RA & 145 & 174 & 268 & 277 & 309 & 379 & 447 & 555 & 600 & - & - & - & 33 \\
\hline Myerson, RB & 310 & 321 & 390 & 409 & 466 & 518 & 628 & 753 & 992 & 1152 & 1492 & 1753 & 50 \\
\hline Nash, JF & 231 & 254 & 266 & 286 & 303 & 312 & 344 & 351 & 402 & 411 & 547 & 840 & 44 \\
\hline North, DC & 107 & 121 & 141 & 147 & 157 & 190 & 212 & 215 & 220 & - & - & - & 27 \\
\hline Ostrom, E & 528 & 569 & 589 & 645 & 662 & 775 & 822 & 853 & 921 & 972 & 986 & 1050 & 68 \\
\hline Phelps, ES & 387 & 523 & 553 & 646 & 673 & 697 & 970 & 1018 & 1155 & 1176 & 1369 & 1420 & 57 \\
\hline Prescott, EC & 492 & 526 & 575 & 681 & 751 & 816 & 985 & 1041 & 1061 & 1077 & 1315 & 1482 & 67 \\
\hline
\end{tabular}


María Isabel Dorta-González, Pablo Dorta-González Evaluación de la trayectoria investigadora ...

\begin{tabular}{|c|c|c|c|c|c|c|c|c|c|c|c|c|c|}
\hline \multirow[b]{2}{*}{ Autor } & \multicolumn{13}{|c|}{$\mathrm{N}-5$} \\
\hline & $\mathrm{H}_{1}$ & $\mathrm{H}_{2}$ & $\mathrm{H}_{3}$ & $\mathrm{H}_{4}$ & $\mathrm{H}_{5}$ & $\mathrm{H}_{6}$ & $\mathrm{H}_{7}$ & $\mathrm{H}_{8}$ & $\mathrm{H}_{9}$ & $\mathrm{H}_{10}$ & $\mathrm{H}_{11}$ & $\mathrm{H}_{12}$ & $\mathrm{I}_{1}$ \\
\hline Samuelson, PA & 41 & 58 & 72 & 83 & 89 & - & - & - & - & - & - & - & 17 \\
\hline Schelling, TC & 91 & 162 & 174 & 239 & 339 & 357 & 367 & 413 & - & - & - & - & 21 \\
\hline Scholes, MS & 109 & 158 & 473 & 617 & 670 & 744 & 800 & 1165 & 1604 & - & - & - & 29 \\
\hline Schultz, TW & 74 & 90 & 95 & 118 & 177 & 200 & - & - & - & - & - & - & 24 \\
\hline Selten, R & 43 & 59 & 92 & 122 & - & - & - & - & - & - & - & - & 19 \\
\hline Sen, AK & 234 & 275 & 430 & 435 & 445 & 450 & 488 & - & - & - & - & - & 48 \\
\hline Sharpe, WF & 79 & 90 & 142 & 397 & 614 & 1096 & - & - & - & - & - & - & 24 \\
\hline Simon, HA & 287 & 303 & 345 & 527 & 595 & 618 & 630 & 673 & 718 & 740 & 770 & 782 & 49 \\
\hline Smith, VL & 505 & 566 & 587 & 607 & 660 & 711 & 760 & 779 & 835 & 876 & 915 & 972 & 65 \\
\hline Solow, RM & 467 & 485 & 517 & 575 & 588 & 611 & 698 & 725 & 776 & 798 & 824 & 858 & 67 \\
\hline Spence, $\mathrm{M}$ & 170 & 204 & 234 & 251 & 354 & 535 & 543 & 621 & 693 & 734 & - & - & 35 \\
\hline Stigler, GJ & 218 & 229 & 257 & 274 & 321 & 342 & 354 & 395 & 441 & 480 & 539 & 660 & 42 \\
\hline Stiglitz, JE & 2066 & 2150 & 2192 & 2273 & 2353 & 2431 & 2471 & 2504 & 2606 & 2638 & 2702 & 2765 & 134 \\
\hline Stone, $\mathrm{R}$ & 58 & 66 & 77 & 103 & 109 & 119 & - & - & - & - & - & - & 18 \\
\hline Tobin, $\mathrm{J}$ & 120 & 137 & 215 & 247 & 289 & 320 & 362 & 379 & 413 & - & - & - & 32 \\
\hline Vickrey, W & 79 & 87 & 112 & 186 & 319 & - & - & - & - & - & - & - & 27 \\
\hline Williamson, $\mathrm{OE}$ & 700 & 868 & 937 & 1048 & 1091 & 1213 & 1270 & 1523 & 1591 & 1927 & 1984 & 2134 & 76 \\
\hline \multirow[b]{2}{*}{ Autor } & \multicolumn{11}{|c|}{$\mathrm{N}-5$} & & \\
\hline & $\mathrm{I}_{2}$ & $\mathrm{I}_{3}$ & $\mathrm{I}_{4}$ & $\mathrm{I}_{5}$ & $\mathrm{I}_{6}$ & $\mathrm{I}_{7}$ & $\mathrm{I}_{8}$ & $\mathrm{I}_{9}$ & $\mathrm{I}_{10}$ & $\mathrm{I}_{11}$ & $\mathrm{I}_{12}$ & & \\
\hline Akerlof, GA & 89 & 120 & 150 & 186 & 225 & 266 & 310 & 362 & 420 & 492 & 564 & & \\
\hline Allais, $\mathrm{M}$ & 82 & 162 & - & - & - & - & - & - & - & - & - & & \\
\hline Arrow, KJ & 54 & 76 & 103 & 130 & 163 & 201 & 250 & 322 & - & - & - & & \\
\hline Aumann, RJ & 95 & 134 & 174 & 224 & 276 & 339 & 403 & 473 & 580 & 760 & 947 & & \\
\hline Becker, GS & 149 & 210 & 274 & 339 & 412 & 484 & 559 & 653 & 745 & 844 & 957 & & \\
\hline Buchanan, JM & 226 & 318 & 412 & 506 & 602 & 699 & 796 & 894 & 992 & 1089 & 1185 & & \\
\hline Coase, $\mathrm{RH}$ & 58 & 87 & 129 & 647 & - & - & - & - & - & - & - & & \\
\hline Debreu, G & 63 & 90 & 112 & 134 & 166 & 202 & 242 & 317 & 396 & 476 & - & & \\
\hline Engle, RF & 138 & 196 & 260 & 323 & 385 & 447 & 510 & 577 & 654 & 743 & 834 & & \\
\hline Fogel, RW & 45 & 64 & 82 & 105 & 138 & 172 & 204 & - & - & - & - & & \\
\hline Friedman, $\mathrm{M}$ & 45 & 104 & - & - & - & - & - & - & - & - & - & & \\
\hline Granger, CWJ & 128 & 178 & 225 & 277 & 335 & 393 & 456 & 527 & 594 & 662 & 730 & & \\
\hline Haavelmo, T & 38 & 59 & 96 & 165 & - & - & - & - & - & - & - & & \\
\hline Harsanyi, JC & 69 & 98 & 133 & 172 & 212 & 254 & 298 & 350 & 445 & 547 & - & & \\
\hline Heckman, JJ & 133 & 183 & 241 & 304 & 364 & 424 & 484 & 544 & 623 & 701 & 784 & & \\
\hline Hurwicz, L & 65 & 97 & 127 & 157 & 241 & 337 & 452 & 582 & - & - & - & & \\
\hline Kahneman, D & 202 & 291 & 382 & 472 & 564 & 655 & 747 & 840 & 937 & 1042 & 1147 & & \\
\hline Klein, LR & 51 & 71 & 93 & 119 & 146 & 177 & 217 & - & - & - & - & & \\
\hline Koopmans, TC & 32 & 46 & 66 & 89 & - & - & - & - & - & - & - & & \\
\hline
\end{tabular}


María Isabel Dorta-González, Pablo Dorta-González Evaluación de la trayectoria investigadora...

\begin{tabular}{|c|c|c|c|c|c|c|c|c|c|c|c|}
\hline \multirow[b]{2}{*}{ Autor } & \multicolumn{11}{|c|}{ N-5 } \\
\hline & $\mathrm{I}_{2}$ & $\mathrm{I}_{3}$ & $\mathrm{I}_{4}$ & $\mathrm{I}_{5}$ & $\mathrm{I}_{6}$ & $\mathrm{I}_{7}$ & $\mathrm{I}_{8}$ & $\mathrm{I}_{9}$ & $\mathrm{I}_{10}$ & $\mathrm{I}_{11}$ & $I_{12}$ \\
\hline Krugman, PR & 150 & 209 & 270 & 331 & 393 & 455 & 518 & 583 & 646 & 715 & 784 \\
\hline Kydland, FE & 56 & 82 & 111 & 143 & 180 & 222 & 306 & 865 & 1505 & - & - \\
\hline Leontief, W & 24 & 37 & 55 & - & - & - & - & - & - & - & - \\
\hline Lucas, RE & 128 & 180 & 237 & 295 & 349 & 400 & 451 & 502 & 553 & 608 & 665 \\
\hline Maskin, ES & 32 & 51 & 72 & 93 & 120 & - & - & - & - & - & - \\
\hline McFadden, DL & 56 & 81 & 104 & 129 & 174 & 266 & - & - & - & - & - \\
\hline Merton, RC & 102 & 144 & 184 & 224 & 264 & 307 & 352 & 406 & 461 & 554 & 656 \\
\hline Miller, MH & 59 & 81 & 109 & 186 & 272 & 379 & 540 & 703 & 1177 & - & - \\
\hline Mirrlees, JA & 70 & 100 & 131 & 161 & 221 & 290 & 366 & 458 & 668 & 895 & 1139 \\
\hline Modigliani, F & 108 & 153 & 197 & 247 & 300 & 358 & 416 & 480 & 552 & 656 & 763 \\
\hline Mundell, RA & 55 & 88 & 122 & 161 & 217 & 295 & 426 & 600 & - & - & - \\
\hline Myerson, RB & 81 & 115 & 149 & 187 & 230 & 285 & 357 & 467 & 608 & 832 & 1141 \\
\hline Nash, JF & 74 & 104 & 134 & 163 & 192 & 224 & 255 & 297 & 339 & 446 & 840 \\
\hline North, DC & 44 & 63 & 82 & 101 & 127 & 158 & 188 & 220 & - & - & - \\
\hline Ostrom, E & 113 & 157 & 203 & 246 & 295 & 346 & 398 & 453 & 510 & 566 & 627 \\
\hline Phelps, ES & 103 & 150 & 202 & 255 & 307 & 385 & 466 & 560 & 654 & 779 & 912 \\
\hline Prescott, EC & 110 & 155 & 205 & 257 & 312 & 380 & 451 & 521 & 589 & 685 & 804 \\
\hline Samuelson, PA & 31 & 48 & 68 & 89 & - & - & - & - & - & - & - \\
\hline Schelling, TC & 42 & 64 & 99 & 159 & 225 & 296 & 413 & - & - & - & - \\
\hline Scholes, MS & 53 & 119 & 207 & 302 & 414 & 544 & 855 & 1604 & - & - & - \\
\hline Schultz, TW & 42 & 59 & 82 & 132 & 200 & - & - & - & - & - & - \\
\hline Selten, $\mathrm{R}$ & 35 & 64 & 122 & - & - & - & - & - & - & - & - \\
\hline Sen, AK & 90 & 162 & 234 & 307 & 380 & 488 & - & - & - & - & - \\
\hline Sharpe, WF & 42 & 70 & 181 & 399 & 1096 & - & - & - & - & - & - \\
\hline Simon, HA & 82 & 117 & 164 & 215 & 267 & 318 & 372 & 430 & 490 & 554 & 617 \\
\hline Smith, VL & 110 & 155 & 199 & 244 & 291 & 340 & 389 & 439 & 491 & 545 & 603 \\
\hline Solow, RM & 110 & 153 & 198 & 240 & 281 & 328 & 374 & 424 & 476 & 530 & 588 \\
\hline Spence, $\mathrm{M}$ & 60 & 87 & 113 & 154 & 231 & 309 & 413 & 553 & 734 & - & - \\
\hline Stigler, GJ & 69 & 95 & 122 & 153 & 186 & 219 & 259 & 309 & 370 & 457 & 660 \\
\hline Stiglitz, JE & 223 & 312 & 401 & 491 & 581 & 671 & 754 & 838 & 922 & 1006 & 1091 \\
\hline Stone, $\mathrm{R}$ & 30 & 44 & 65 & 88 & 119 & - & - & - & - & - & - \\
\hline Tobin, $\mathrm{J}$ & 53 & 83 & 117 & 157 & 203 & 260 & 322 & 413 & - & - & - \\
\hline Vickrey, W & 45 & 70 & 130 & 319 & - & - & - & - & - & - & - \\
\hline Williamson, $\mathrm{OE}$ & 132 & 189 & 250 & 311 & 377 & 442 & 520 & 599 & 697 & 794 & 899 \\
\hline
\end{tabular}




\begin{tabular}{|c|c|c|c|c|c|c|c|c|c|c|c|c|c|}
\hline \multirow[b]{2}{*}{ Autor } & \multicolumn{13}{|c|}{$\mathrm{N}$} \\
\hline & $\mathrm{H}_{1}$ & $\mathrm{H}_{2}$ & $\mathrm{H}_{3}$ & $\mathrm{H}_{4}$ & $\mathrm{H}_{5}$ & $\mathrm{H}_{6}$ & $\mathrm{H}_{7}$ & $\mathrm{H}_{8}$ & $\mathrm{H}_{9}$ & $\mathrm{H}_{10}$ & $\mathrm{H}_{11}$ & $\mathrm{H}_{12}$ & $\mathrm{I}_{1}$ \\
\hline Akerlof, GA & 543 & 563 & 652 & 701 & 763 & 804 & 814 & 886 & 929 & 1057 & 1213 & 1390 & 68 \\
\hline Allais, M & 103 & 128 & 241 & - & - & - & - & - & - & - & - & - & 43 \\
\hline Arrow, KJ & 236 & 248 & 302 & 421 & 438 & 461 & 494 & 511 & 555 & 559 & 569 & 592 & 44 \\
\hline Aumann, RJ & 475 & 489 & 532 & 559 & 767 & 815 & 838 & 909 & 1082 & 1092 & 1375 & 1462 & 59 \\
\hline Becker, GS & 807 & 867 & 961 & 1136 & 1384 & 1458 & 1483 & 1521 & 1632 & 1758 & 1818 & 2161 & 87 \\
\hline Buchanan, JM & 2449 & 2496 & 2635 & 2726 & 2772 & 2860 & 2988 & 3114 & 3198 & 3242 & 3324 & 3366 & 146 \\
\hline Coase, RH & 94 & 106 & 165 & 229 & 280 & 297 & 971 & - & - & - & - & - & 28 \\
\hline Debreu, G & 276 & 309 & 359 & 394 & 425 & 451 & 514 & 556 & 576 & 612 & 714 & 732 & 48 \\
\hline Engle, RF & 1151 & 1179 & 1265 & 1406 & 1794 & 1871 & 1943 & 1962 & 2101 & 2142 & 2314 & 2353 & 97 \\
\hline Fogel, RW & 117 & 144 & 150 & 181 & 205 & 233 & 240 & 241 & - & - & - & - & 33 \\
\hline Friedman, $\mathrm{M}$ & 78 & 95 & 125 & 178 & 232 & 337 & 353 & - & - & - & - & - & 24 \\
\hline Granger, CWJ & 1082 & 1198 & 1340 & 1392 & 1470 & 1520 & 1643 & 1687 & 1821 & 1862 & 1965 & 2083 & 92 \\
\hline Haavelmo, T & 47 & 104 & 117 & 153 & 196 & - & - & - & - & - & - & - & 19 \\
\hline Harsanyi, JC & 246 & 281 & 323 & 342 & 407 & 437 & 512 & 520 & 531 & 551 & 652 & 664 & 48 \\
\hline Heckman, JJ & 1017 & 1101 & 1154 & 1257 & 1281 & 1326 & 1437 & 1500 & 1518 & 1615 & 1803 & 1838 & 93 \\
\hline Hurwicz, L & 130 & 238 & 263 & 285 & 293 & 309 & 611 & 632 & 678 & 689 & - & - & 31 \\
\hline Kahneman, D & 2206 & 2250 & 2506 & 2586 & 2911 & 3148 & 3217 & 3284 & 3460 & 3919 & 4051 & 4076 & 138 \\
\hline Klein, LR & 147 & 189 & 204 & 218 & 241 & 252 & 262 & 272 & 298 & - & - & - & 35 \\
\hline Koopmans, TC & 92 & 102 & 106 & 117 & 122 & 151 & 166 & - & - & - & - & - & 26 \\
\hline Krugman, PR & 1323 & 1387 & 1415 & 1501 & 1700 & 1806 & 2042 & 2061 & 2104 & 2193 & 2255 & 2291 & 107 \\
\hline Kydland, FE & 205 & 297 & 317 & 342 & 356 & 422 & 467 & 531 & 792 & 1916 & 2043 & - & 35 \\
\hline Leontief, W & 92 & 116 & 119 & 125 & 130 & 143 & 147 & - & - & - & - & - & 26 \\
\hline Lucas, RE & 897 & 978 & 1161 & 1210 & 1234 & 1258 & 1493 & 1528 & 1541 & 1571 & 1704 & 1710 & 87 \\
\hline Maskin, ES & 118 & 128 & 136 & 157 & 160 & 166 & 173 & - & - & - & - & - & 28 \\
\hline McFadden, DL & 116 & 143 & 224 & 231 & 257 & 266 & 288 & 376 & - & - & - & - & 32 \\
\hline Merton, RC & 582 & 683 & 699 & 785 & 809 & 999 & 1017 & 1089 & 1104 & 1288 & 1308 & 1781 & 72 \\
\hline Miller, MH & 224 & 245 & 275 & 313 & 347 & 546 & 776 & 841 & 970 & 1088 & 1363 & 1471 & 44 \\
\hline Mirrlees, JA & 250 & 271 & 302 & 360 & 402 & 463 & 676 & 761 & 811 & 903 & 1368 & 1394 & 46 \\
\hline Modigliani, F & 413 & 495 & 509 & 685 & 822 & 910 & 955 & 974 & 1061 & 1203 & 1242 & 1249 & 56 \\
\hline Mundell, RA & 155 & 184 & 279 & 312 & 334 & 400 & 669 & 723 & 754 & - & - & - & 35 \\
\hline Myerson, RB & 390 & 436 & 508 & 575 & 638 & 763 & 865 & 919 & 1008 & 1100 & 1480 & 1664 & 60 \\
\hline Nash, JF & 458 & 477 & 512 & 544 & 573 & 614 & 640 & 664 & 698 & 729 & 748 & 774 & 62 \\
\hline North, DC & 166 & 196 & 215 & 222 & 235 & 241 & 282 & 292 & 318 & 326 & 332 & - & 36 \\
\hline Ostrom, E & 1112 & 1199 & 1226 & 1252 & 1330 & 1381 & 1430 & 1455 & 1611 & 1719 & 1762 & 1821 & 97 \\
\hline Phelps, ES & 417 & 453 & 502 & 637 & 722 & 763 & 825 & 856 & 1074 & 1198 & 1356 & 1642 & 60 \\
\hline Prescott, EC & 687 & 704 & 783 & 799 & 889 & 992 & 1072 & 1147 & 1217 & 1239 & 1364 & 1515 & 71 \\
\hline Samuelson, PA & 99 & 113 & 126 & 138 & 153 & 169 & 177 & 184 & - & - & - & - & 29 \\
\hline Schelling, TC & 121 & 353 & 360 & 365 & 469 & 499 & 527 & 680 & - & - & - & - & 30 \\
\hline Scholes, MS & 143 & 198 & 352 & 627 & 773 & 800 & 932 & 972 & 1505 & 2231 & - & - & 35 \\
\hline
\end{tabular}




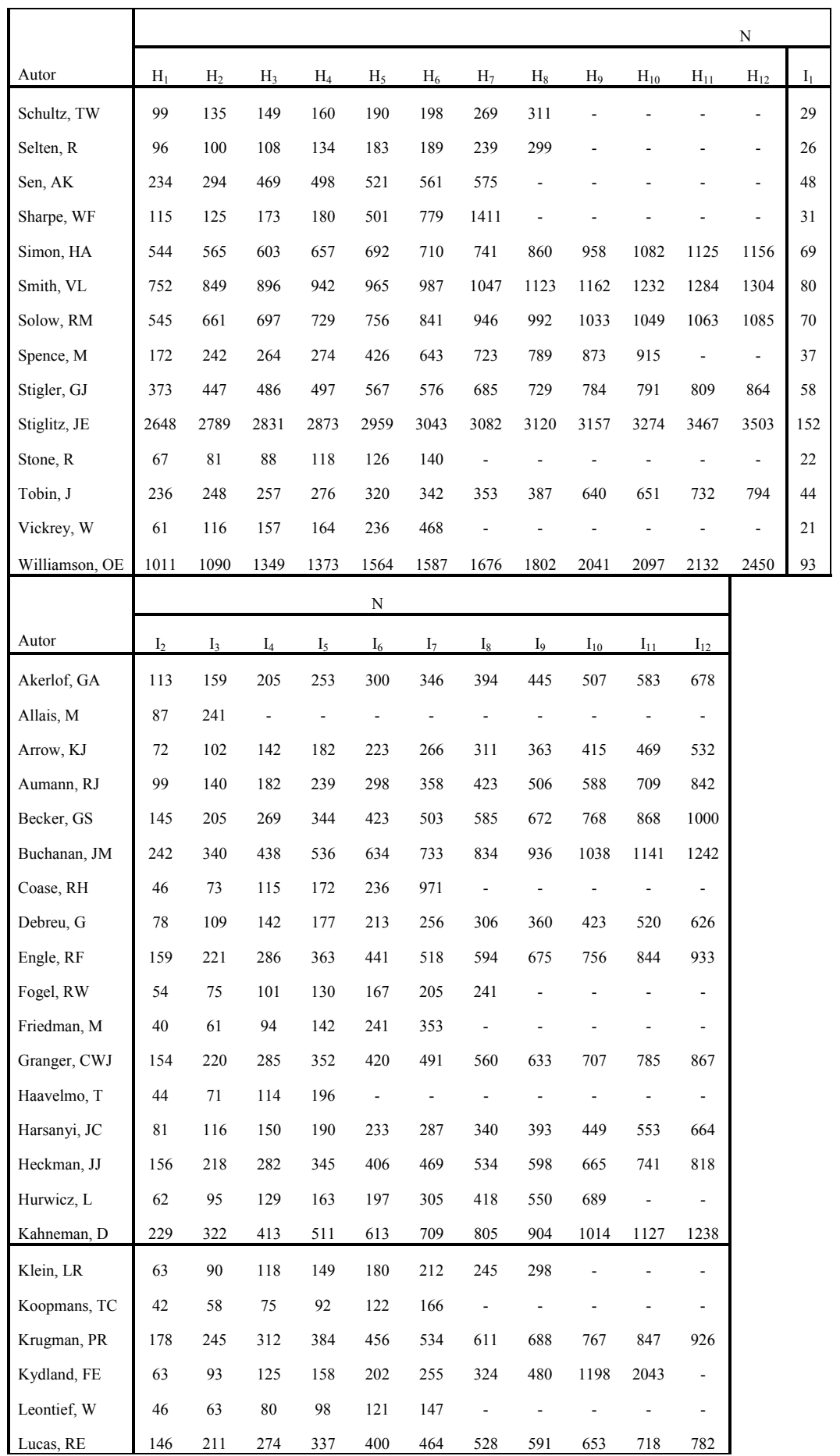


María Isabel Dorta-González, Pablo Dorta-González Evaluación de la trayectoria investigadora ...

\begin{tabular}{|c|c|c|c|c|c|c|c|c|c|c|c|}
\hline \multirow[b]{2}{*}{ Autor } & \multicolumn{11}{|c|}{$\mathrm{N}$} \\
\hline & $\mathrm{I}_{2}$ & $\mathrm{I}_{3}$ & $\mathrm{I}_{4}$ & $\mathrm{I}_{5}$ & $\mathrm{I}_{6}$ & $\mathrm{I}_{7}$ & $\mathrm{I}_{8}$ & $\mathrm{I}_{9}$ & $\mathrm{I}_{10}$ & $\mathrm{I}_{11}$ & $\mathrm{I}_{12}$ \\
\hline Maskin, ES & 48 & 68 & 91 & 114 & 140 & 173 & - & - & - & - & - \\
\hline McFadden, DL & 53 & 84 & 115 & 152 & 190 & 239 & 376 & - & - & - & - \\
\hline Merton, RC & 123 & 174 & 225 & 276 & 339 & 401 & 469 & 537 & 624 & 713 & 869 \\
\hline Miller, MH & 69 & 95 & 124 & 155 & 210 & 296 & 391 & 510 & 656 & 893 & 1183 \\
\hline Mirrlees, JA & 73 & 102 & 135 & 170 & 211 & 280 & 361 & 451 & 564 & 832 & 1113 \\
\hline Modigliani, F & 95 & 134 & 181 & 237 & 298 & 361 & 424 & 494 & 579 & 668 & 757 \\
\hline Mundell, RA & 58 & 93 & 132 & 174 & 232 & 379 & 552 & 754 & - & - & - \\
\hline Myerson, RB & 100 & 144 & 191 & 242 & 303 & 370 & 439 & 518 & 608 & 760 & 944 \\
\hline Nash, JF & 103 & 144 & 184 & 223 & 263 & 304 & 345 & 388 & 432 & 476 & 522 \\
\hline North, DC & 61 & 87 & 110 & 133 & 156 & 186 & 217 & 254 & 292 & 332 & - \\
\hline Ostrom, E & 163 & 227 & 290 & 355 & 421 & 487 & 553 & 624 & 699 & 774 & 849 \\
\hline Phelps, ES & 101 & 142 & 189 & 241 & 295 & 352 & 406 & 480 & 566 & 670 & 814 \\
\hline Prescott, EC & 116 & 163 & 210 & 259 & 312 & 368 & 427 & 489 & 550 & 620 & 701 \\
\hline Samuelson, PA & 47 & 66 & 86 & 108 & 133 & 158 & 184 & - & - & - & - \\
\hline Schelling, TC & 77 & 125 & 173 & 247 & 331 & 429 & 680 & - & - & - & - \\
\hline Scholes, MS & 62 & 107 & 189 & 293 & 400 & 536 & 684 & 1095 & 2231 & - & - \\
\hline Schultz, TW & 51 & 74 & 96 & 124 & 152 & 213 & 311 & - & - & - & - \\
\hline Selten, $\mathrm{R}$ & 40 & 53 & 70 & 99 & 129 & 184 & 299 & - & - & - & - \\
\hline Sen, AK & 94 & 173 & 258 & 349 & 457 & 575 & - & - & - & - & - \\
\hline Sharpe, WF & 50 & 77 & 105 & 239 & 510 & 1411 & - & - & - & - & - \\
\hline Simon, HA & 115 & 161 & 209 & 257 & 304 & 351 & 404 & 463 & 532 & 603 & 676 \\
\hline Smith, VL & 136 & 192 & 249 & 305 & 360 & 417 & 477 & 538 & 602 & 668 & 732 \\
\hline Solow, RM & 121 & 170 & 217 & 261 & 309 & 361 & 416 & 472 & 529 & 586 & 645 \\
\hline Spence, $\mathrm{M}$ & 66 & 96 & 124 & 176 & 271 & 384 & 519 & 696 & 915 & - & - \\
\hline Stigler, GJ & 97 & 135 & 173 & 215 & 256 & 307 & 361 & 420 & 479 & 539 & 608 \\
\hline Stiglitz, JE & 251 & 347 & 443 & 539 & 635 & 730 & 824 & 917 & 1012 & 1111 & 1209 \\
\hline Stone, $\mathrm{R}$ & 37 & 52 & 76 & 102 & 140 & - & - & - & - & - & - \\
\hline Tobin, J & 72 & 97 & 123 & 152 & 181 & 209 & 242 & 324 & 408 & 518 & 657 \\
\hline Vickrey, W & 44 & 76 & 108 & 174 & 468 & - & - & - & - & - & - \\
\hline Williamson, $\mathrm{OE}$ & 154 & 224 & 293 & 368 & 443 & 521 & 602 & 692 & 783 & 874 & 978 \\
\hline
\end{tabular}


María Isabel Dorta-González, Pablo Dorta-González Evaluación de la trayectoria investigadora...

Tabla III. Correlaciones entre indicadores y diferencias.

\begin{tabular}{|c|c|c|c|c|c|c|c|c|c|c|c|c|c|}
\hline & & \multicolumn{12}{|c|}{$\mathrm{N}-5$} \\
\hline & & $\mathrm{H}_{1}$ & $\mathrm{H}_{2}$ & $\mathrm{H}_{3}$ & $\mathrm{H}_{4}$ & $\mathrm{H}_{5}$ & $\mathrm{H}_{6}$ & $\mathrm{H}_{7}$ & $\mathrm{H}_{8}$ & $\mathrm{H}_{9}$ & $\mathrm{H}_{10}$ & $\mathrm{H}_{11}$ & $\mathrm{H}_{12}$ \\
\hline \multirow{12}{*}{ z } & $\mathrm{H}_{1}$ & 0,986 & & & & & & & & & & & \\
\hline & $\mathrm{H}_{2}$ & 0,984 & 0,982 & & & & & & & & & & \\
\hline & $\mathrm{H}_{3}$ & 0,982 & 0,984 & 0,98 & & & & & & & & & \\
\hline & $\mathrm{H}_{4}$ & 0,981 & 0,984 & 0,984 & 0,983 & & & & & & & & \\
\hline & $\mathrm{H}_{5}$ & 0,971 & 0,979 & 0,981 & 0,984 & 0,974 & & & & & & & \\
\hline & $\mathrm{H}_{6}$ & 0,965 & 0,973 & 0,977 & 0,982 & 0,977 & 0,973 & & & & & & \\
\hline & $\mathrm{H}_{7}$ & 0,94 & 0,947 & 0,952 & 0,964 & 0,977 & 0,984 & 0,977 & & & & & \\
\hline & $\mathrm{H}_{8}$ & 0,963 & 0,971 & 0,977 & 0,98 & 0,983 & 0,985 & 0,979 & 0,972 & & & & \\
\hline & $\mathrm{H}_{9}$ & 0,94 & 0,952 & 0,968 & 0,973 & 0,977 & 0,979 & 0,975 & 0,98 & 0,947 & & & \\
\hline & $\mathrm{H}_{10}$ & 0,87 & 0,886 & 0,918 & 0,925 & 0,927 & 0,926 & 0,921 & 0,941 & 0,973 & 0,955 & & \\
\hline & $\mathrm{H}_{11}$ & 0,902 & 0,916 & 0,927 & 0,928 & 0,935 & 0,939 & 0,936 & 0,946 & 0,975 & 0,971 & 0,964 & \\
\hline & $\mathrm{H}_{12}$ & 0,915 & 0,935 & 0,942 & 0,942 & 0,95 & 0,958 & 0,961 & 0,969 & 0,972 & 0,969 & 0,97 & 0,953 \\
\hline & Media & 0,95 & 0,956 & 0,961 & 0,963 & 0,963 & 0,964 & 0,958 & 0,962 & 0,967 & 0,965 & 0,967 & 0,953 \\
\hline & & \multicolumn{12}{|c|}{$\mathrm{N}-5$} \\
\hline & & $\mathrm{I}_{1}$ & $\mathrm{I}_{2}$ & $\mathrm{I}_{3}$ & $\mathrm{I}_{4}$ & $\mathrm{I}_{5}$ & $\mathrm{I}_{6}$ & $\mathrm{I}_{7}$ & $\mathrm{I}_{8}$ & $\mathrm{I}_{9}$ & $\mathrm{I}_{10}$ & $\mathrm{I}_{11}$ & $\mathrm{I}_{12}$ \\
\hline \multirow{13}{*}{ z } & $\mathrm{I}_{1}$ & 0,977 & & & & & & & & & & & \\
\hline & $\mathrm{I}_{2}$ & 0,976 & 0,974 & & & & & & & & & & \\
\hline & $\mathrm{I}_{3}$ & 0,946 & 0,956 & 0,959 & & & & & & & & & \\
\hline & $\mathrm{I}_{4}$ & 0,962 & 0,968 & 0,968 & 0,952 & & & & & & & & \\
\hline & $\mathrm{I}_{5}$ & 0,93 & 0,941 & 0,955 & 0,966 & 0,734 & & & & & & & \\
\hline & $\mathrm{I}_{6}$ & 0,817 & 0,828 & 0,857 & 0,914 & 0,794 & 0,802 & & & & & & \\
\hline & $\mathrm{I}_{7}$ & 0,427 & 0,445 & 0,48 & 0,595 & 0,842 & 0,978 & 0,937 & & & & & \\
\hline & $\mathrm{I}_{8}$ & 0,77 & 0,784 & 0,819 & 0,845 & 0,872 & 0,893 & 0,9 & 0,88 & & & & \\
\hline & $\mathrm{I}_{9}$ & 0,612 & 0,623 & 0,695 & 0,747 & 0,788 & 0,84 & 0,875 & 0,928 & 0,832 & & & \\
\hline & $\mathrm{I}_{10}$ & 0,199 & 0,208 & 0,308 & 0,393 & 0,459 & 0,532 & 0,591 & 0,753 & 0,964 & 0,827 & & \\
\hline & $\mathrm{I}_{11}$ & 0,384 & 0,364 & 0,372 & 0,377 & 0,392 & 0,409 & 0,421 & 0,471 & 0,844 & 0,913 & 0,909 & \\
\hline & $\mathrm{I}_{12}$ & 0,677 & 0,673 & 0,677 & 0,679 & 0,716 & 0,762 & 0,805 & 0,854 & 0,889 & 0,908 & 0,938 & 0,789 \\
\hline & Media & 0,723 & 0,706 & 0,709 & 0,719 & 0,7 & 0,745 & 0,755 & 0,777 & 0,882 & 0,883 & 0,924 & 0,789 \\
\hline
\end{tabular}


María Isabel Dorta-González, Pablo Dorta-González Evaluación de la trayectoria investigadora...

\begin{tabular}{|c|c|c|c|c|c|c|c|c|c|c|c|c|c|}
\hline & & \multicolumn{12}{|c|}{$\mathrm{N}-5$} \\
\hline & & \multicolumn{12}{|c|}{ diferencia de correlaciones (entorno-intervalo) } \\
\hline \multirow{12}{*}{$\mathrm{z}$} & & 0,009 & & & & & & & & & & & \\
\hline & & 0,008 & 0,008 & & & & & & & & & & \\
\hline & & 0,036 & 0,028 & 0,021 & & & & & & & & & \\
\hline & & 0,02 & 0,017 & 0,016 & 0,031 & & & & & & & & \\
\hline & & 0,041 & 0,038 & 0,027 & 0,019 & 0,24 & & & & & & & \\
\hline & & 0,149 & 0,145 & 0,12 & 0,069 & 0,183 & 0,171 & & & & & & \\
\hline & & 0,513 & 0,502 & 0,472 & 0,369 & 0,136 & 0,005 & 0,04 & & & & & \\
\hline & & 0,193 & 0,187 & 0,157 & 0,135 & 0,111 & 0,092 & 0,079 & 0,092 & & & & \\
\hline & & 0,327 & 0,329 & 0,273 & 0,226 & 0,189 & 0,139 & 0,1 & 0,052 & 0,115 & & & \\
\hline & & 0,671 & 0,678 & 0,611 & 0,532 & 0,468 & 0,395 & 0,329 & 0,189 & 0,009 & 0,129 & & \\
\hline & & 0,518 & 0,552 & 0,556 & 0,551 & 0,542 & 0,53 & 0,515 & 0,475 & 0,131 & 0,058 & 0,056 & \\
\hline & & 0,238 & 0,262 & 0,265 & 0,263 & 0,234 & 0,196 & 0,156 & 0,115 & 0,083 & 0,061 & 0,031 & 0,164 \\
\hline & Suma & 2,723 & 2,747 & 2,519 & 2,195 & 2,103 & 1,528 & 1,219 & 0,923 & 0,338 & 0,247 & 0,087 & 0,164 \\
\hline
\end{tabular}

Tabla IV. Indicadores para N-5.

\begin{tabular}{|c|l|c|c|c|}
\hline & Autor & $\mathrm{H}=\mathrm{h}^{2}$ & $\mathrm{H}_{\mathrm{h} / 2}$ & $\mathrm{H}_{\mathrm{h} / 2} / \mathrm{H}$ \\
\hline 1 & Akerlof, GA & 361 & 785 & 2,2 \\
2 & Allais, M & 16 & 120 & 7,5 \\
3 & Arrow, KJ & 100 & 222 & 2,2 \\
4 & Aumann, RJ & 256 & 809 & 3,2 \\
5 & Becker, GS & 324 & 1381 & 4,3 \\
6 & Buchanan, JM & 2025 & 3710 & 1,8 \\
7 & Coase, RH & 36 & 135 & 3,8 \\
8 & Debreu, G & 144 & 311 & 2,2 \\
9 & Engle, RF & 676 & 1901 & 2,8 \\
10 & Fogel, RW & 81 & 134 & 1,7 \\
11 & Friedman, M & 16 & 61 & 3,8 \\
12 & Granger, CWJ & 729 & 1677 & 2,3 \\
13 & Haavelmo, T & 36 & 93 & 2,6 \\
14 & Harsanyi, JC & 144 & 372 & 2,6 \\
15 & Heckman, JJ & 529 & 1449 & 2,7 \\
16 & Hurwicz, L & 100 & 253 & 2,5 \\
17 & Kahneman, D & 1369 & 3744 & 2,7 \\
18 & Klein, LR & 81 & 157 & 1,9 \\
19 & Koopmans, TC & 36 & 66 & 1,8 \\
20 & Krugman, PR & 841 & 2064 & 2,5 \\
21 & Kydland, FE & 121 & 303 & 2,5 \\
22 & Leontief, W & 25 & 38 & 1,5 \\
\hline
\end{tabular}




\begin{tabular}{|c|l|c|c|c|}
\hline & Autor & $\mathrm{H}^{2} \mathrm{~h}^{2}$ & $\mathrm{H}_{\mathrm{h} / 2}$ & $\mathrm{H}_{\mathrm{h} / 2} / \mathrm{H}$ \\
\hline 23 & Lucas, RE & 576 & 1237 & 2,1 \\
24 & Maskin, ES & 49 & 102 & 2,1 \\
25 & McFadden, DL & 64 & 173 & 2,7 \\
26 & Merton, RC & 324 & 838 & 2,6 \\
27 & Miller, MH & 121 & 541 & 4,5 \\
28 & Mirrlees, JA & 169 & 575 & 3,4 \\
29 & Modigliani, F & 256 & 766 & 3,0 \\
30 & Mundell, RA & 100 & 309 & 3,1 \\
31 & Myerson, RB & 225 & 628 & 2,8 \\
32 & Nash, JF & 169 & 312 & 1,8 \\
33 & North, DC & 100 & 157 & 1,6 \\
34 & Ostrom, E & 484 & 986 & 2,0 \\
35 & Phelps, ES & 289 & 1018 & 3,5 \\
36 & Prescott, EC & 361 & 1061 & 2,9 \\
37 & Samuelson, PA & 36 & 72 & 2,0 \\
38 & Schelling, TC & 81 & 239 & 3,0 \\
39 & Scholes, MS & 100 & 670 & 6,7 \\
40 & Schultz, TW & 49 & 95 & 1,9 \\
41 & Selten, R & 25 & 59 & 2,4 \\
42 & Sen, AK & 64 & 435 & 6,8 \\
43 & Sharpe, WF & 49 & 142 & 2,9 \\
44 & Simon, HA & 256 & 673 & 2,6 \\
45 & Smith, VL & 484 & 915 & 1,9 \\
46 & Solow, RM & 324 & 776 & 2,4 \\
47 & Spence, M & 121 & 354 & 2,9 \\
48 & Stigler, GJ & 169 & 342 & 2,0 \\
49 & Stiglitz, JE & 1936 & 3846 & 2,0 \\
50 & Stone, R & 49 & 77 & 1,6 \\
51 & Tobin, J & 100 & 289 & 2,9 \\
52 & Vickrey, W & 36 & 112 & 3,1 \\
53 & Williamson, OE & 676 & 2572 & 3,8 \\
\hline & & & & \\
\hline
\end{tabular}

